\title{
Quantification of image quality of intra-fractional cone-beam computed tomography for arc irradiation with various imaging condition
}

\author{
Masahide Saito, Yuki Shibata, Koji Ueda, Hidekazu Suzuki, Takafumi Komiyama, Kan Marino, \\ Shinichi Aoki, Yoshiyasu Maehata, Naoki Sano, Hiroshi Onishi \\ Department of Radiology, University of Yamanashi, Yamanashi, Japan
}

\begin{abstract}
Background: 3-dimensional intra-cone beam computed tomography (intra-CBCT) could be a potentially powerful tool for use with arc irradiation such as volumetric modulated arc therapy. The aim of the study was to evaluate the image quality of intra-cone beam computed tomography (intra-CBCT) for arc irradiation with various imaging condition.

Materials and methods: Two types of intra-CBCT imaging techniques were evaluated - intra-fractional CBCT with flattening filtered (FF) beam (intra-FF CBCT) and that with flattening filter free (FFF) beam (intra-FFF CBCT). For the intra-MV beams, four different field sizes $(2 \mathrm{~cm} \times 2 \mathrm{~cm}, 5 \mathrm{~cm} \times 5 \mathrm{~cm}, 10 \mathrm{~cm} \times 10 \mathrm{~cm}$, and $20 \mathrm{~cm} \times 20 \mathrm{~cm}$ ) were used with dose rates of $500 \mathrm{MU} / \mathrm{min}$ and $1600 \mathrm{MU} / \mathrm{min}$, for $6 \mathrm{MVFF}$ and $6 \mathrm{MV} \mathrm{FFF}$, respectively. For all image acquisitions, two rotation angles (full-arc and half-arc) were investigated. Thereafter, the linearity, contrast-to-noise ratio (CNR), and uniformity index (UI) of intra-CBCT image were compared with those of conventional CBCT image.

Results: All acquisition conditions had good linearity of the $C T$ value $\left(R^{2}>0.99\right)$. For $C N R$, the change rates from conventional CBCT ranged from $0.6-33.7 \%$ for a $2 \mathrm{~cm} \times 2 \mathrm{~cm}$ beam, whereas that for a $20 \mathrm{~cm} \times 20 \mathrm{~cm}$ beam ranged from $62.7-82.3 \%$. Similarly, the UI increased from $1.5 \%$ to $7.0 \%$ as the field size increased.

Conclusion: Quality of intra-CBCT image was affected by the field size and acquisition angle. Image quality of intra-CBCT was worse than that of conventional $\mathrm{CBCT}$, but it was better under a smaller field and wider correction angle and would be acceptable for clinical use.

Key words: cone-beam computed tomography; volumetric modulated arc therapy; MV simultaneous exposure; image quality Rep Pract Oncol Radiother 2021;26(3):495-501
\end{abstract}

\section{Introduction}

In high conformal radiotherapy for a tumor with respiratory motion, monitoring the intra-fractional tumor motion is important to confirm the accuracy of irradiation and reduce internal margin. Institutes globally have used multiple techniques to monitor tumor motion during irradiation. One of the techniques involves the use of an electronic portal imaging device (EPID), which enables to monitor the target localization using the treatment beam on cine mode EPID image [1]. Another technique is a 4-dimensional (4-D) kV-cone-beam computed tomography

Address for correspondence: Masahide Saito, Ph.D., Department of Radiology, University of Yamanashi, 1110 Shimokato, Chuo-city, Yamanashi, 409-3898, Japan, tel: +81-55-273-1111, fax: +81-55-273-6744; e-mail: masahides@yamanashi.ac.jp

This article is available in open access under Creative Common Attribution-Non-Commercial-No Derivatives 4.0 International (CC BY-NC-ND 4.0) license, allowing to download articles and share them with others as long as they credit the authors and the publisher, but without permission to change them in any way or use them commercially 
(CBCT). This technique enables to verify the tumor position and other anatomical positions during treatment to generate the respiratory correlated CBCT by projection phase sorting [2]. Some studies have investigated the effectiveness and improvement of $4 \mathrm{D}-\mathrm{kV} \mathrm{CBCT}$ to assess the respiratory motion of tumor during high conformal treatments, such as volumetric modulated arc therapy (VMAT) [3-6].

In this study, we investigated the effectiveness of intra-fractional CBCT, called intra-CBCT, a potentially powerful tool for use with arc irradiation such as volumetric modulated arc therapy. Although the quality of intra-CBCT images is worse due to the ray scattering derived from MV beam irradiation and the acquisition of them gives an extra dose, no study has evaluated the details of image quality during arc irradiation.

\section{Materials and methods}

\section{Equipment for intra-CBCT}

$\mathrm{X}$-ray volume imaging $\left(\mathrm{XVI}^{\circ}\right)$ system ver. 5.0 (Elekta AB, Crawley, UK) attached to the Elekta Synergy was used in this study. It utilizes an amorphous silicon/cesium iodide flat-panel detector with a $1024 \times 1024$ array of $0.4-\mathrm{mm}$ elements. The X-ray source is mounted on a retractable arm with a fixed source to an isocenter distance of $100 \mathrm{~cm}$ and offers several different interchangeable collimators and $\mathrm{kV}$ filter combinations based on the desired scanning volume. For acquiring intra-CBCT images, the $3 \mathrm{D}$ Volume View $^{\mathrm{TM}}$ mode was used, which are reconstructed automatically after the acquisition of projection images. For reconstruction of intra-CBCT images, beam delivery has to be accompanied by a rotational treatment, such as conformal arc radiotherapy and VMAT.

In this study, three types of CBCT images were compared, namely: 1) conventional CBCT image, 2) intra-CBCT image acquired using a $6 \mathrm{MV}$ flattening filter ( $\mathrm{FF}$ ) beam (intra-FF CBCT), and 3) intra-CBCT image acquired using a $6 \mathrm{MV}$ with flattening filter free (FFF) beam (intra-FFF CBCT). Two collection angles (full-arc: CW, $-180^{\circ}$ to $180^{\circ}$ and half-arc: $\mathrm{CW},-180^{\circ}$ to $20^{\circ}$ ) were investigated for image acquisition. All images were acquired with a $120-\mathrm{kV}, 20-\mathrm{mA}$ with the following imaging parameters: S10 collimator $(27 \mathrm{~cm}$ of ax- ial field-of-view (FOV), $12 \mathrm{~cm}$ of nominal axial length); image size, $540 \times 540$ pixels; pixel size, 0.5 $\mathrm{mm}$; and slice thickness, $0.5 \mathrm{~mm}$. For the intra-MV beams, the monitor unit (MU) was set as 1000, and four different field sizes $(2 \mathrm{~cm} \times 2 \mathrm{~cm}, 5 \mathrm{~cm} \times 5 \mathrm{~cm}$, $10 \mathrm{~cm} \times 10 \mathrm{~cm}$, and $20 \mathrm{~cm} \times 20 \mathrm{~cm}$ ) were used with dose rates of $500 \mathrm{MU} / \mathrm{min}$ and $1600 \mathrm{MU} / \mathrm{min}$, for 6 MV FF and 6 MV FFF, respectively.

\section{Evaluation of image quality}

The Catphan ${ }^{\circledR} 503$ (The Phantom Laboratory, Salem, NY, US) phantom with a $20-\mathrm{cm}$ diameter was used for the evaluation of intra-CBCT image quality. It has the following different inserts for evaluating the geometric accuracy with defined distances in the axial and longitudinal directions: 1 ) a section with uniform material and a density equivalent to that of water for determining spatial uniformity; 2) a section featuring a cylindrically arranged resolution pattern in an axial plane (1 through 21 line pairs per centimeter) and two point markers (tungsten-carbide bead; diameter, $0.28 \mathrm{~mm}$ ) embedded into a uniform material; and 3) a section with inserts of different densities such as polystyrene, polymethylpentene (PMP), low-density polyethylene (LDPE), acrylic, air, Delrin, and Teflon implemented for conversion testing of electron density to the CT number. The phantom was set in the treatment couch top and the laser was aligned as shown in Figure 1A.

Image quality evaluation was performed using the following three indexes: 1) linearity between nominal and measured CT values; 2) contrast-to-noise ratio (CNR); and 3) uniformity index (UI). For the linearity, the average CT value was measured with nine regions of interest (ROIs), where each material existed (two materials had water). For the CNR, the value was calculated by following equation (1):

$$
C N R=\frac{S_{m a t}-S_{B . G .}}{\sigma_{B . G .}}
$$

where, $S_{\text {mat }}$ is the mean pixel value of each material; $S_{B . G \text {. }}$ is the mean pixel value of the background (water), and $\sigma_{B . G \text {. }}$ is the standard deviation of the background (water). Figure $1 \mathrm{~B}$ shows the image slice used for evaluation of the linearity and CNR. 

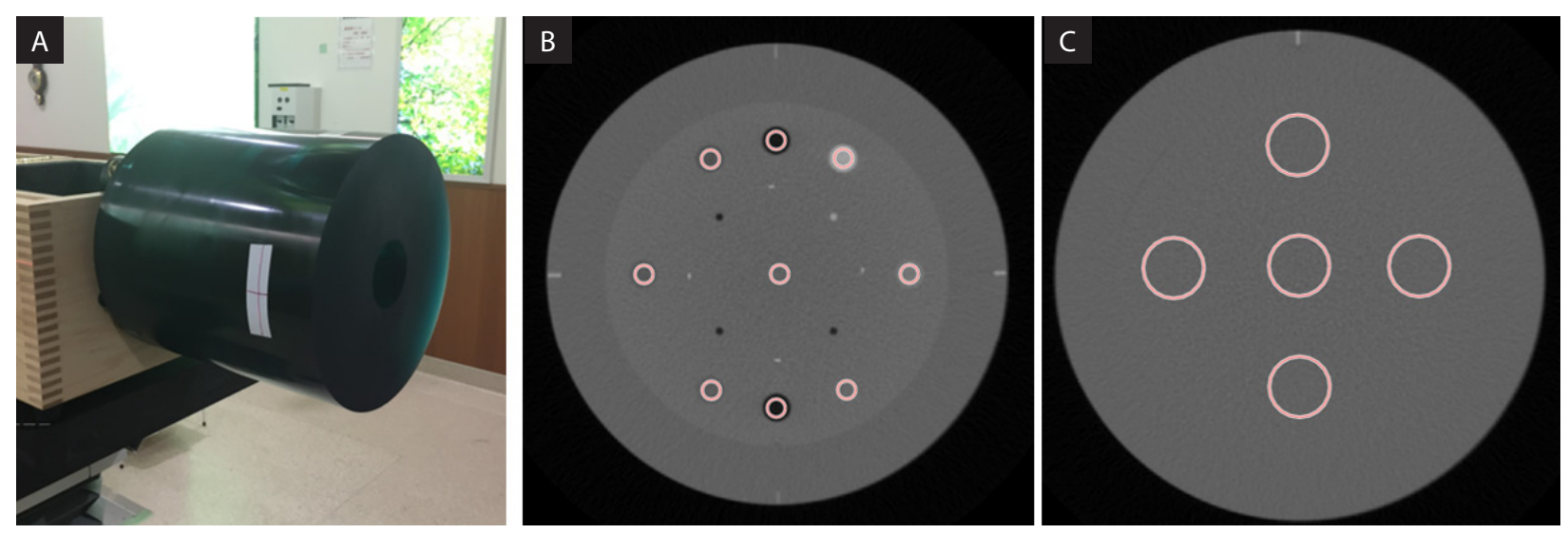

Figure 1. Setup of the Catphan ${ }^{\circledR 5} 503$ phantom in the head of treatment couch (A), and the region of interest (ROI) for each image analysis (B, C); B - the image slice used for evaluating the linearity and contrast-to-noise ratio (CNR), which has nine ROIs with each material; $\mathbf{C}$ — the slice for the evaluation of the uniformity index (UI) which has five homogeneity ROls

To assess the UI, the average CT value was measured with five ROIs at regions with uniformity. Then, the UI was calculated with the following equation (2):

$$
U I=100 \times \frac{\overline{H U}_{\text {maximum }}-\overline{H U}_{\text {center }}}{\overline{H U}_{\text {center }}+1000}
$$

where, $\overline{H U}_{\text {maximum }}$ is the mean $\mathrm{HU}$ that is the largest value among the peripheral four ROIs; $\overline{H U}_{\text {center }}$ is the mean $\mathrm{HU}$ of the center of the ROI. Figure 1C shows the image slice used for the evaluation of UI.

All analyses were performed using MATLAB R2016a (Mathworks Inc., Natick, MA).

\section{Results}

Figure 2 shows the comparison among three $\mathrm{CBCT}$ images (conventional, intra-FF, and intra-FFF CBCT images with a field size of $10 \mathrm{~cm} \times 10$ $\mathrm{cm})$. The slice which has a 1 through 21-line pair per centimeter high-resolution test gauge is shown for each image. On comparison between conventional and intra-CBCT images, the latter contained more noise than the former because of MV beam scattering during the imaging of the phantom. Especially, intra-FFF CBCT contained more noise than intra-FF CBCT. Furthermore, on focusing on the collection angles, full-arc showed better image recognition than those acquired with half-arc. Figure 3 also shows the lateral profiles of each CBCT image with the same condition of Figure 2, indicating that intra-CBCT images contain noise from $\mathrm{MV}$ beam scattering. Figure 4 shows the comparison among four field sizes (intra-CBCT with the field size of $2 \mathrm{~cm} \times 2 \mathrm{~cm}, 5 \mathrm{~cm} \times 5 \mathrm{~cm}, 10 \mathrm{~cm} \times 10 \mathrm{~cm}$, and $20 \mathrm{~cm} \times 20 \mathrm{~cm}$ ) for two collection angles. The image quality was worse as the field size increased. Thereafter, all CBCT images were quantitatively analyzed. Table 1 shows the linearity between the nominal and measured CT values. Good linearity was observed for both conventional $\mathrm{CBCT}$ and intra-CBCT (FF and FFF) images $\left(\mathrm{R}^{2}>0.99\right.$ for all acquisition conditions). Figure 5 shows the change in CNR values depending on each field size for seven materials. CNR values decreased as the field size increased for all conditions. Table 2 shows the average value of the $\mathrm{CNR}$ change rate (\%) for all materials assessed using conventional CBCT images for each condition. The change rates from conventional CBCT ranged from $0.6-33.7 \%$ for a $2 \mathrm{~cm} \times 2 \mathrm{~cm}$ beam, whereas that ranged from $62.7-82.3 \%$ for a $20 \mathrm{~cm} \times 20 \mathrm{~cm}$ beam. With regard to the collection angles, full-arc showed better CNR than the half-arc. Similarly, for beam quality, intra-FF CBCT images showed better CNR than intra-FFF CBCT images. Figure 6 shows the changes in UI depending on each field size for the intra-FF and intra-FFF CBCT images with two collection angles. For all conditions, the UI increased as the field sizes increased (approximately, from -1.5 to 7.0 ).

\section{Discussion}

Quality assurance of the $\mathrm{kV}$-CBCT system is one of the important topics in radiotherapy and new research, such as image quality of dual en- 


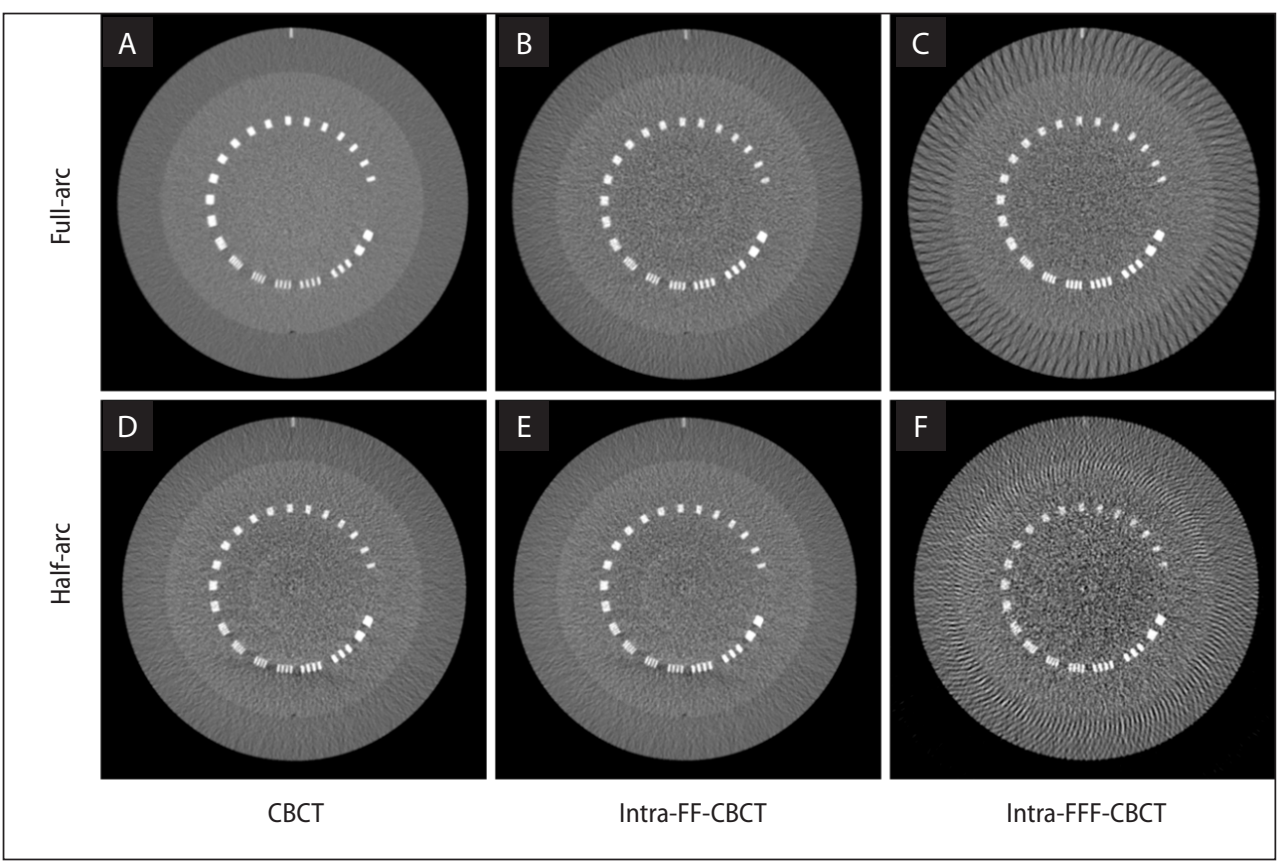

Figure 2. Comparison of three CBCT images $[(\mathbf{A})$ and (D), conventional $C B C T$ images; (B) and (E), intra-FF CBCT images; and $(\mathbf{C})$ and $(\mathbf{F})$, intra-FFF CBCT images] at the slice which has a 1 through 21-line pair per centimeter high-resolution test gauge. Two collection angles (upper, full-arc; bottom, half-arc) are shown. The image size is $540 \times 540$ pixels, and the pixel size was $0.5 \mathrm{~mm}$ for all dimensions. Window width of $1,000 \mathrm{HU}$ and window level of $10 \mathrm{HU}$ are shown

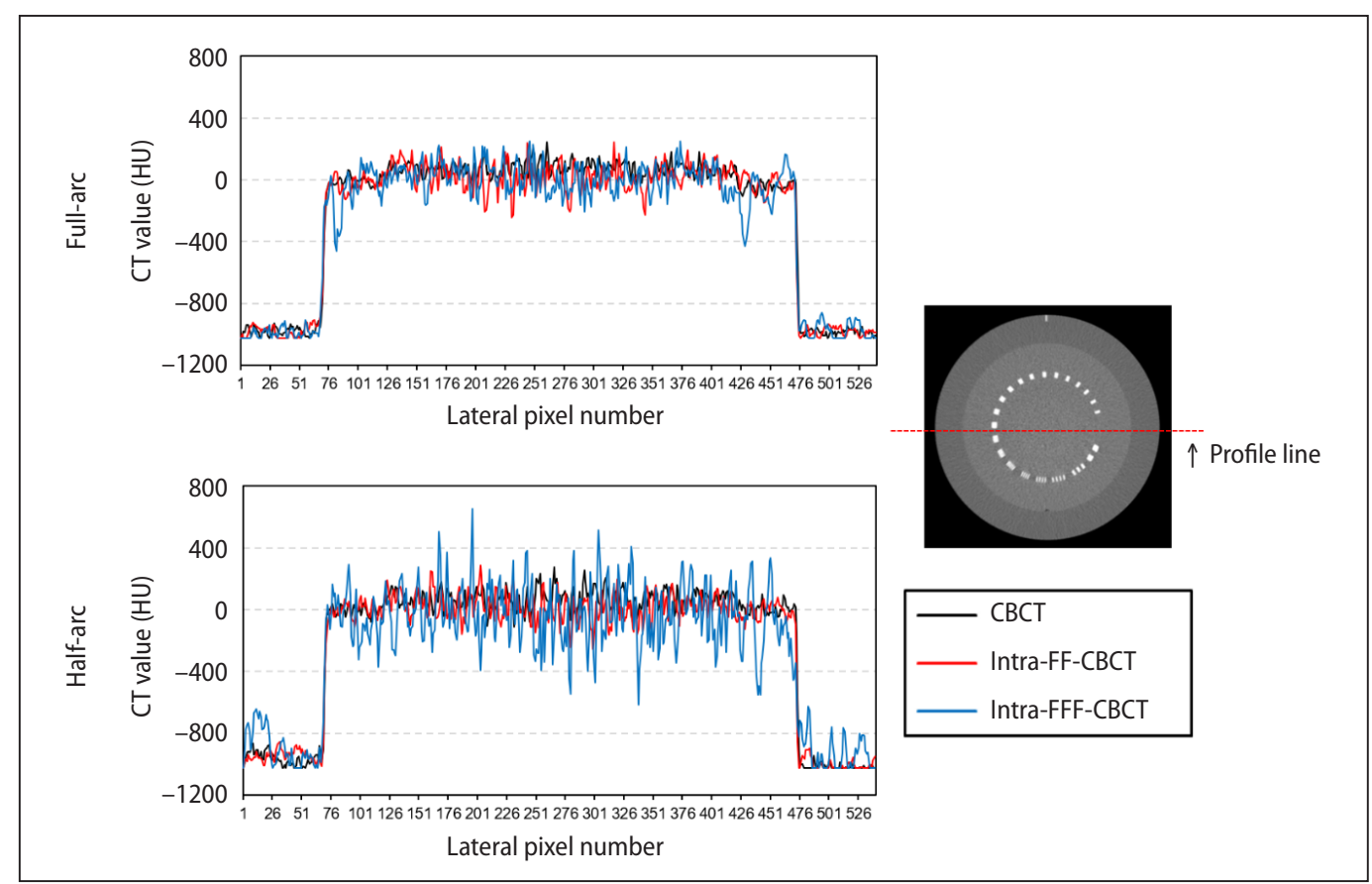

Figure 3. Lateral profiles of three CBCT images (black line, conventional CBCT images; red line, intra-FF CBCT images; and blue line, intra-FFF CBCT images). Two collection angles (upper, full-arc; bottom, half-arc) are shown. The selected profile line is shown in right figure (red line)

ergy CT, has been carried out [7]. However, in our knowledge, there has been no reports to investi- gate the image quality of intra-CBCT with various imaging conditions in detail. In our results, image 


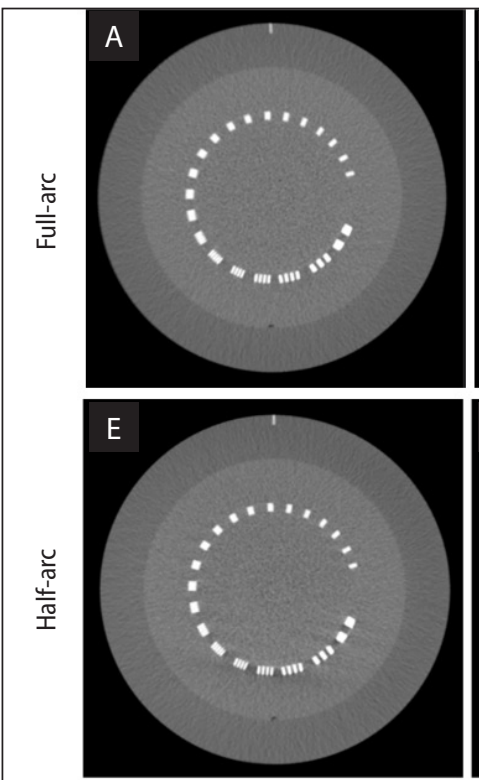

$2 \mathrm{~cm} \times 2 \mathrm{~cm}$
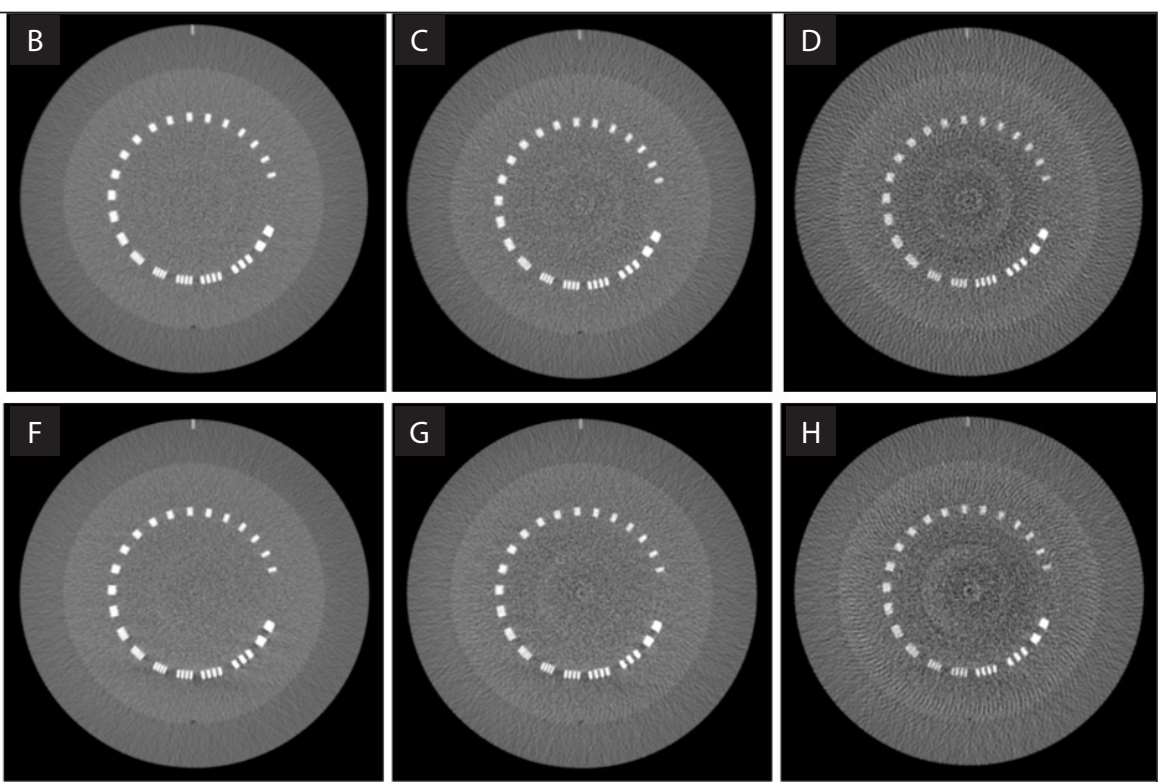

$5 \mathrm{~cm} \times 5 \mathrm{~cm}$

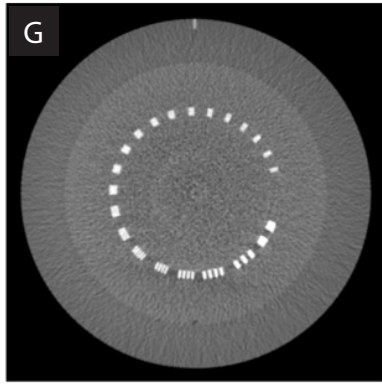

$10 \mathrm{~cm} \times 10 \mathrm{~cm}$

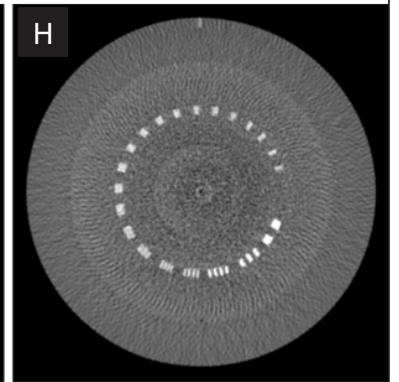

$20 \mathrm{~cm} \times 20 \mathrm{~cm}$

Figure 4. Comparison among four field sizes [(A) and (E), $2 \mathrm{~cm} \times 2 \mathrm{~cm}$; (B) and (F), $5 \mathrm{~cm} \times 5 \mathrm{~cm}$; (C) and (G), $10 \mathrm{~cm} \times 10 \mathrm{~cm}$; and (D) and (H), $20 \mathrm{~cm} \times 20 \mathrm{~cm}$ ] at the slice which has a 1 through 21-line pair per centimeter high-resolution test gauge. The intra FF-CBCT images with two collection angles (upper, full-arc; bottom, half-arc) are shown. The image size is $540 \times 540$ pixels, and the pixel size was $0.5 \mathrm{~mm}$ for all dimensions. Window width of $1,000 \mathrm{HU}$ and window level of $10 \mathrm{HU}$ are shown

Table 1. Linearity $\left(R^{2}\right)$ between the nominal and measured CT values

\begin{tabular}{|l|l|c|c|c|c|c|}
\cline { 3 - 7 } & \multicolumn{6}{c}{ Linearity $\left(R^{2}\right)$} \\
\hline \multirow{2}{*}{ FF } & Full-arc & 0.9999 & 0.9999 & 0.9999 & 0.9998 & 0.9996 \\
\cline { 2 - 7 } & Half-arc & 0.9995 & 0.9996 & 0.9997 & 0.9993 & 0.9996 \\
\hline \multirow{2}{*}{ FFF } & Full-arc & 0.9999 & 0.9998 & 0.9999 & 0.9999 & 0.9991 \\
\cline { 2 - 7 } & Half-arc & 0.9995 & 0.9994 & 0.9990 & 0.9995 & 0.9968 \\
\hline
\end{tabular}

quality depended on the field size and acquisition angle. With regard to field size, the scattered dose from the subject increased when the field size was large, which resulted in deteriorating image quality. With regard to the acquisition angle, full-arc imaging provided better image quality than half-arc because the former usually generates more projection images during treatment. Therefore, our study suggested that treatment with small fields, such as stereotactic body radiotherapy, might be preferred to use intra-CBCT. However, this study did not perform the evaluation with actual patient images and, therefore, additional study is required.

Our study had some limitations. First, we investigated the image quality by only one vender
(Elekta XVI). Our results might differ from those on other vendor machines. Second, our study only investigated images acquired with the following condition: $120 \mathrm{kV}$ and $20 \mathrm{~mA}$ with $\mathrm{S}$ collimator. Therefore, different future studies should investigate image acquisition with different irradiation conditions. Third, in this study, we only evaluated the following two beam qualities: $6 \mathrm{MV}$ FF and 6 MV FFF with the same MU. From our results, the quality of images acquired with FF seems better than acquired with FFF. However, the dose rate and gantry speed between them differed, indicating that they cannot be compared with each other. Therefore, further studies are needed to compare different beam qualities. 


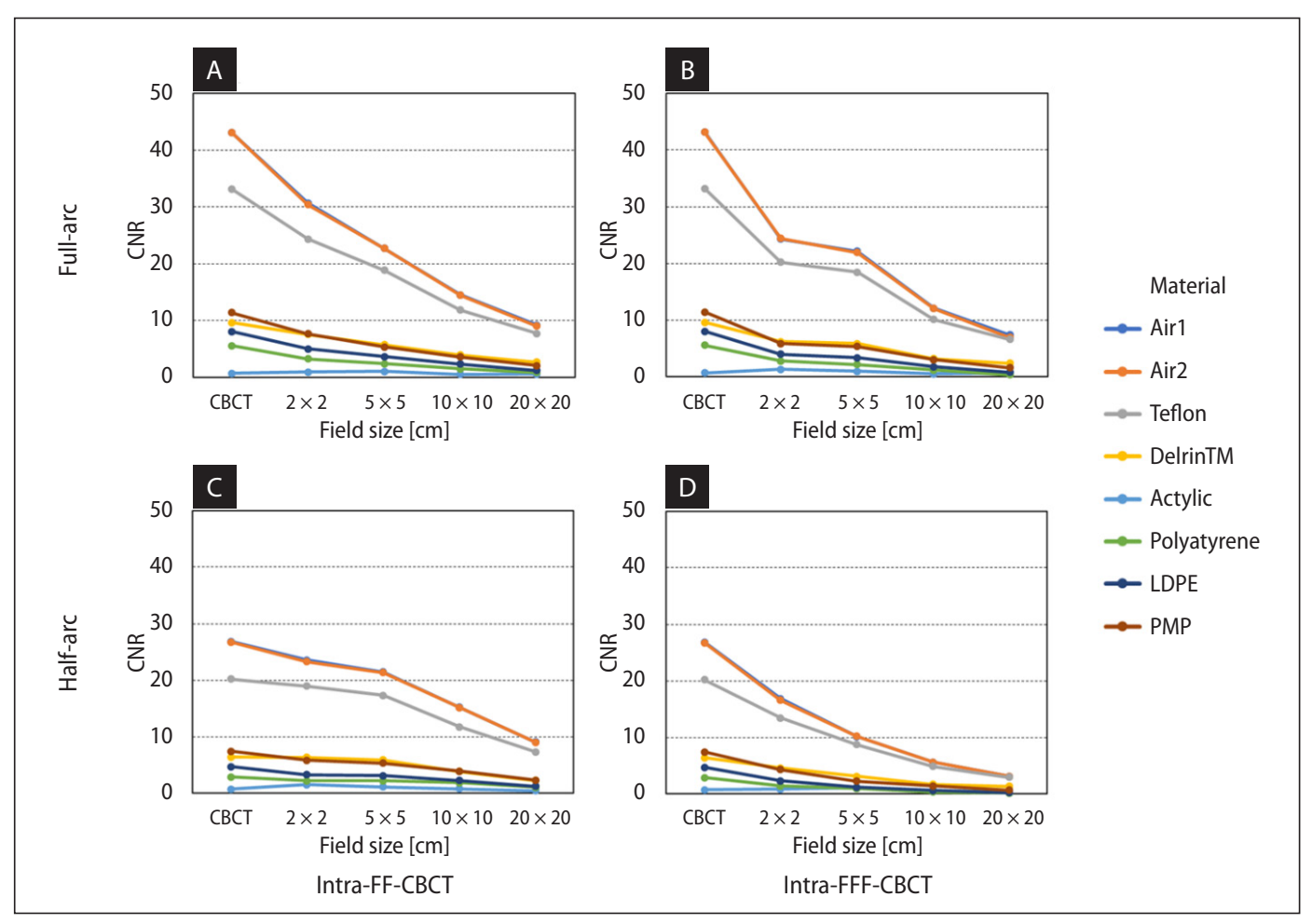

Figure 5. Changes in contrast-to-noise ratio depending on the field size $(2 \mathrm{~cm} \times 2 \mathrm{~cm}, 5 \mathrm{~cm} \times 5 \mathrm{~cm}, 10 \mathrm{~cm} \times 10 \mathrm{~cm}$, $20 \mathrm{~cm} \times 20 \mathrm{~cm}$ ) for seven materials. (A) and (C) show the results of intra-FF CBCT images and (B) and (D) show the results of intra-FFF-CBCT images. Two collection angles (upper, full-arc; bottom, half-arc) are shown

Table 2. The average value of the change rate (\%) of contrast-to-noise ratio for all materials as evaluated using conventional CBCT images for each condition

\begin{tabular}{l|l|c|c|c|c|}
\cline { 3 - 5 } \multicolumn{2}{c}{} & \multicolumn{5}{c}{ CNR change rate (\%) } \\
\hline \multirow{3}{*}{ Full-arc } & FF & $2 \mathrm{~cm} \times 2 \mathrm{~cm}$ & $5 \mathrm{~cm} \times 5 \mathrm{~cm}$ & $10 \mathrm{~cm} \times 10 \mathrm{~cm}$ & $20 \mathrm{~cm} \times 20 \mathrm{~cm}$ \\
\cline { 2 - 6 } & FFF & $-23.9 \pm 20.7$ & $-36.9 \pm 32.8$ & $-62.1 \pm 14.2$ & $-72.3 \pm 20.5$ \\
\hline \multirow{2}{*}{ Half-arc } & FF & $-27.1 \pm 44.8$ & $-38.6 \pm 32.3$ & $-66.3 \pm 18.0$ & $-74.6 \pm 26.7$ \\
\cline { 2 - 6 } & FFF & $-0.6 \pm 39.5$ & $-11.8 \pm 25.7$ & $-37.1 \pm 16.4$ & $-62.7 \pm 10.3$ \\
\hline
\end{tabular}

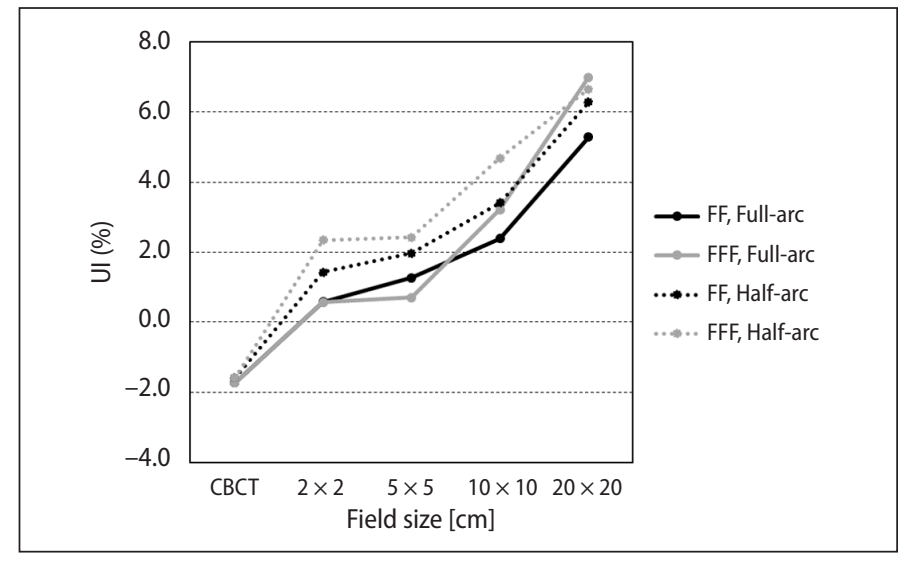

Figure 6. Uniformity index changes depending on the field size $(2 \mathrm{~cm} \times 2 \mathrm{~cm}, 5 \mathrm{~cm} \times 5 \mathrm{~cm}, 10 \mathrm{~cm} \times 10 \mathrm{~cm}, 20 \mathrm{~cm} \times 20 \mathrm{~cm})$. The intra-FF CBCT (black line) and intra-FF CBCT (gray line) for two collection angles [full-arc (solid line) and half-arc (dash line)] are shown 


\section{Conclusion}

In this study, we evaluated intra $\mathrm{CBCT}$ at the various imaging conditions using the Catphan ${ }^{\oplus} 503$ phantom. Quality of intra-CBCT image was affected by the field size and acquisition angle. Image quality of intra-CBCT was worse than that of conventional CBCT, but it was better under a smaller field and wider correction angle and would be acceptable for clinical use.

\section{Conflicts of interest}

There is no conflict of interest with regard to this manuscript.

None declared.

\section{Funding}

\section{Reference}

1. Berbeco RI, Hacker F, lonascu D, et al. Clinical feasibility of using an EPID in CINE mode for imageguided verification of stereotactic body radiotherapy. Int J Radiat Oncol Biol Phys. 2007; 69(1): 258-266, doi: 10.1016/j.ijrobp.2007.04.051, indexed in Pubmed: 17707280.

2. Sonke JJ, Zijp L, Remeijer P, et al. Respiratory correlated cone beam CT. Med Phys. 2005; 32(4): 1176-1186, doi: 10.1118/1.1869074, indexed in Pubmed: 15895601.

3. Nakagawa K, Yamashita H, Shiraishi K, et al. Verification of in-treatment tumor position using kilovoltage cone-beam computed tomography: a preliminary study. Int J Radiat Oncol Biol Phys. 2007; 69(4): 970-973, doi: 10.1016/j. ijrobp.2007.08.026, indexed in Pubmed: 17967297.

4. Ling $C$, Zhang P, Etmektzoglou T, et al. Acquisition of MV-scatter-free kilovoltage CBCT images during RapidArc ${ }^{\text {TM }}$ or VMAT. Radiother Oncol. 2011; 100(1): 145-149, doi: 10.1016/j.radonc.2011.07.010, indexed in Pubmed: 21821301.

5. Nakagawa K, Haga A, Shiraishi K, et al. First clinical conebeam CT imaging during volumetric modulated arc therapy. Radiother Oncol. 2009; 90(3): 422-423, doi: 10.1016/j. radonc.2008.11.009, indexed in Pubmed: 19062117.

6. Kida S, Masutani $Y$, Yamashita $H$, et al. In-treatment 4D cone-beam CT with image-based respiratory phase recognition. Radiol Phys Technol. 2012; 5(2): 138-147, doi: 10.1007/s12194-012-0146-5, indexed in Pubmed: 22367851.

7. Pawałowski B, Szweda H, Dudkowiak A, et al. Quality evaluation of monoenergetic images generated by dualenergy computed tomography for radiotherapy: A phantom study. Phys Med. 2019; 63: 48-55, doi: 10.1016/j. ejmp.2019.05.019, indexed in Pubmed: 31221408. 